\title{
Old-Age Pension and Extended Families: How is Adult Children's Internal Migration Affected?
}

\author{
Xi Chen \\ School of Public Health and Department of Economics, Yale University
}

\begin{abstract}
This paper makes use of the most recent social pension reform in rural China to examine whether receipt of the pension payment equips adult children of pensioners to migrate. Employing a regression discontinuity (hereafter $\mathrm{RD}$ ) design to a primary longitudinal survey, this paper overcomes challenges in the literature that households eligible for pension payment might be systematically different from ineligible households and that it is difficult to separate the effect of pension from that of age or cohort heterogeneity. Around the pension eligibility age cut-off, results reveal large and significant increase among adult sons (but not daughters) to migrate out of their home county. Meanwhile, adult children are more likely to migrate out if their parents are healthy. Our Fuzzy RD estimations survive a standard set of key placebo tests and robustness checks.
\end{abstract}

\section{Keywords}

Rural Pension; RD Design; Adult Children; Migration

\section{Introduction}

Old-age pension programs targeting the elderly may eventually benefit their extended families. However, no consensus has been reached on the growing body of literature that examines the potential impact of old-age pension on migration decisions of extended families. Bertrand et al. (2003) find no salient increase in migration among prime-age members of the household living with the pensioners. Taking non-resident members of the household (including those who left to find work) into consideration, Posel et al. (2006) find pension income significantly promotes female labor migration, while there is no impact on male labor. Ardington et al. (2009) and Sun and Eggleston (2014) show that the rate of migration increases for both male and female prime-aged labor due to pension receipt, while Jensen (2004) finds no such effect.

Migration promotes individual well-being. Despite the large wage gap, institutional, economic and cultural barriers hinder people living in rural areas from migrating to urban areas to find better jobs. This issue is of first-order importance for China as a world factory but faced with labor shortage. In the meantime, China's population ages rapidly. By 2050, over 25.6 percent of China's population is expected to be over age $65 .{ }^{1}$ Moreover, the implementation of stringent fertility policy in the last three decades has dramatically driven 
up the dependency ratio at a relatively low income level, which further intensifies the motivation among China's vast albeit shrinking working-age population to stay at home villages and take care of their elderly parents.

In late 2009, China launched the New Rural Pension Scheme (NRPS hereafter) for rural residents, now covering more than 300 million Chinese. This program offers a unique setting for studying the ageing population, given the rapidity of China's population ageing, traditions of filial piety, decreasing number of children, and dearth of formal social security, at a relatively low income level. Different from the most generous pension program in South Africa that pays twice the average income to all women above age 55 and all men above age 60 (Lund 2007), the program in our surveyed region pays all elders above age 60 around 25 percent of per capita income. This paper examines whether this pension payment to elderly parents encourages their adult children to migrate from their home counties to pursue better lives.

Distinguished from most of the existing strand of literature, we make use of a discontinuous jump in pension receipt due to differences in the age of the elder(s) in the households around the pension eligibility age cut-off. This empirical strategy may overcome two main challenges. First, People eligible for pension payment can be systematically different from those ineligible. Controlling for individual fixed effects in panel data estimations, such as Ardington et al. (2009), requires very specific assumptions about the nature of unobserved factors and their persistence over time. Meanwhile, individuals who dropped out of the demographic surveillance between waves of the survey cannot be followed, which generates biases in longitudinal estimations. The RD design compares people who fall within a narrow bandwidth around the cut-off age to isolate the causal impact of pension receipt from potential systematic differences between people eligible for pension and those ineligible. Second, granted according to age, the prior research examining the link between old-age pension and migration decisions of adult children cannot separate the effect of pension receipt from that of life-cycle pattern or cohort heterogeneity of elderly parents. Assuming that children's migration decision is continuous with respect to the age of the elderly, the RD design, already applied to multiple health policy settings (e.g. Edmonds et al. 2005; Card et al. 2009; Miller et al. 2013; Sun and Eggleston 2014), is able to disentangle the effect of pension receipt from other age-related factors that shape migration decisions.

Adult children lend major support to their parents in rural communities and therefore might be more sensitive to parents' receiving pension payment. Therefore, our sample for empirical analysis is composed of families with adult children and at least one parent. We draw on a rich survey to track all adult children's migration status and demographic information, regardless of whether she lives with parents, so we are able to link parents with non-resident adult children to study the migration pattern for all adult children. We can also generate datasets of pensioners' and non-pensioners' adult children without introducing complication of endogenous household formation. Though our survey is not nationally representative, we believe this study has methodological advantages and the results make it of broad interest.

${ }^{1}$ Source: Population Division of the Department of Economic and Social Affairs of the United Nations Secretariat, World Population Prospects: The 2010 Revision, http://esa.un.org/unpd/wpp/index.htm. 
In the patriarchal rural society adult sons are more responsible to take care of parents, while adult daughters tend to migrate when they are married. Our results utilizing a longitudinal household survey reveal a large and significant increase in adult son's migration rate around the cut-off age for pension received by their parents, while there is no such effect for adult daughters. Overall, receiving pension payment increases adult children's migration rate by 28.7 percentage points. Considering that 43.2 percent of adult children migrate before their parents become eligible for pension payment, the NRPS pension increase their rate of migration by two-thirds. Moreover, adult children are more likely to migrate if their parents are healthy.

The RD design survives a series of key placebo tests and robustness checks, including verifying no jumps of outcomes at placebo age cut-offs, no discrete changes in baseline characteristics and predetermined outcomes at the age cut-off, no discontinuity of age density, no confounded policy change at the age cut-off, and that results are robust to various bandwidths, bandwidth selection procedures, and polynomial specifications.

The rest of the paper is organized as follows. Section 2 introduces the new rural pension program in China and its implementation in Guizhou province. Section 3 describes the data and the identification strategy. Section 4 presents results and discusses interpretations and mechanisms. Section 5 conducts a series of key robust checks. Section 6 concludes.

\section{The New Rural Pension Reform in China and Guizhou Province}

The pension system in rural China had largely been vacant until late 2009 when China launched the NRPS, now covering more than 300 million Chinese (Appendix Figure 1). The NRPS provides an opportunity to examine the pension impact when its payment is modest. Both the central government and local governments provide subsidy to pensioners. The local government subsidy must be no less than $30 \mathrm{RMB}$ per person per year. The higher individual premium one chooses to pay, the more subsidies local government pays. The individual premium and the local government subsidy form the individual account. Besides, a basic pension benefit financed by the central government, 55 RMB per month per person in Guizhou but as much as 310 RMB per month per person in a few rich provinces / municipalities, is available to all residents. Pensioners receive payment from both the individual account and the basic pension benefit.

All rural residents aged 16 or above who are not enrolled in urban basic pension program can enroll in NRPS voluntarily. Participants below age 45 are required to contribute at least 15 years to become eligible for pension payment upon reaching age 60 . Those aged between 45 and 60 are encouraged to contribute more to meet the shortfall in contributions to their own account over the years before age 60 . Those aged 60 or above do not have individual accounts as the NRPS was only rolled out recently. Therefore, pension payment is noncontributory to anyone aged 60 or above by the local rollout time of NRPS. In other words, they are not required to make any contributions to the NRPS system, have cumulative work histories, or have extended families to become eligible for the basic pension benefit. ${ }^{2}$ Since the NRPS is not tied to retirement decisions, these decisions should not confound potential impacts of pension receipt around the cutoff age 60 . 
The NRPS may have heterogeneous impacts due to China's large regional disparities. To the rich people with alternative old-age support, their willingness to participate in the pension program can be low. However, the NRPS can be attractive to the poor people with no formal old-age support. While the benefit accounts for a smaller proportion of income in developed regions, it can be much larger in less developed regions. For example, in our surveyed area in Guizhou, pension payment represents one fourth of average income (Appendix Table 1). Considering that the elderly earn less than the young generation, the pension payment should exert a larger impact.

\section{Empirical Strategy}

\subsection{Data}

This paper makes use of a rich panel survey of households in Guizhou province. We attempted to interview all residents in 26 randomly selected villages in a county in Guizhou, one of the poorest provinces in China. With a total population of 402,000 people, per capita income in the county is above the provincial median but below the provincial mean, suggesting that its income profile is representative of Guizhou province as a whole. Albeit double-digit income growth, half of the residents in the 26 villages are still in poverty (Appendix Table 1).

Each wave of survey was conducted a few days before the Lunar New Year (January or February) when most migrants return to celebrate. We also managed to visit each household several times to make sure all family members at home were interviewed individually. In the latest 2011 wave 5189 individuals from 963 households were interviewed. Both the 2006 wave and the 2011 wave survey are utilized in the analysis. Since the NRPS was introduced in Guizhou in early 2010, the 2006 wave is used in placebo tests. ${ }^{3}$

Any potential policies that may invalidate the 2006 wave as a placebo year have to affect the migration patterns of cohorts on two sides of cutoff age (in 2006) differently or affect 2006 and 2011 differently in order to confound the real pension impact. To the best of our knowledge, no such policies exist. Though there might be somewhat stronger signals of labor shortage in 2011, this should not disqualify year 2006 as a placebo year. Frist, no labor market condition in 2006 treated younger and older age cohorts differently. Second, the labor market was tightened in both 2006 and 2011 with similar migration rate around the age cutoff, suggesting that it is not likely to affect the two years differently. ${ }^{4}$

\footnotetext{
${ }^{2}$ Some regions in China stipulate that those aged 60 or above when the NRPS rolled out in the local county are eligible for the basic pension benefit only when their children eligible for the NRPS also enroll in. However, this is not the case in our surveyed region in Guizhou.

${ }^{3}$ The survey has four waves, i.e. 2004, 2006, 2009 and 2011. Each wave was implemented in January and/or February of the following year, i.e. 2005, 2007, 2010 and 2012. Since Guizhou province started the pilot NRPS in early 2010, the 2011 wave is in the post-NRPS period and the first three waves are in the pre-NRPS period. The 2004 wave data is not used in this analysis because many questions are not compatible with the later three waves. Meanwhile, our placebo tests do not use the 2009 wave data out of two concerns. First, people may already have had information on future implementation of the NRPS by the 2009 wave. The resulting anticipation effect may undermine the identification of the pension effect. Second, the 2009 wave overlapped with the recent world financial crisis and therefore might contaminate or bring unknown noises to our identification of the main outcome of interest, i.e. migration status, through other discontinuous changes around the age cut-off. However, placebo tests using the 2009 wave, available upon request, are largely consistent with those using the 2006 wave. In 2009, the overall migration rate for adult children whose parents aged 50-70 was lower than in 2006, because some migrants returned to their home villages in 2009 when they could not find jobs in coastal factories. Fortunately, the quick economic recovery in China brought them back to migrant jobs since early 2010, so our 2011 survey was not affected by this economic and labor market turmoil.
} 
Five subsamples are analyzed in our analysis, including the subsample of adult children ( $\mathrm{N}=1741)$, the subsample of adult son $(\mathrm{N}=1016)$, the subsample of adult daughter $(\mathrm{N}=725)$, the subsample of adult children with a parent in poor health $(\mathrm{N}=970)$, and the subsample of adult children with all parents in good health $(\mathrm{N}=771)$. The survey collected rich information on demographics, income and consumption for all family members (including returning migrants and other non-resident members), their relationship with household head and migration status. Besides, every household was asked by the survey enumerators to show its Hukou book, an official registration record for every family member, to verify family composition.

Migration status was identified from the family roster questionnaire, which records all coresident and non-resident members. Migrants are defined as those who left the home county for at least six months in the past year. Relative to non-migrant children, a majority of migrant children are engaged in more profitable nonfarm work (including manufacturing, construction, public services, etc.) than farming activities (Figure 1).

\subsection{The RD design}

The empirical challenge in obtaining a consistent estimate of the causal effect of pension is that pension receipt is endogenous. We utilize an age discontinuity in the benefit structure of the social pension program. This discontinuity produces an abrupt shift in eligibility among otherwise similar individuals and households. Assuming other household and individual characteristics are smoothly distributed according to age, i.e., individuals barely ineligible for pension payment (in their late 50s) and those barely eligible (in their early 60s) are comparable, the abrupt shift in eligibility allows us to isolate the causal link between parental pension receipt and adult children's migration. Specifically, two assumptions must hold. First, the outcome $y$ for an age a depends on (observed and unobserved) characteristics of the household and all of its potential members that must have smooth effects on the outcome. Second, families cannot respond to the pension policies by altering the age of the elderly.

Social pension income depends on whether one passes age 60. Figure 2A plots the sample means of pension receipt by normalized age, where 0 represents the eligibility threshold of exact age 60. As expected, there is a sharp upward break in pension receipt at the age cutoff.

We focus on the local treatment effect of pension income. Since a few people below age 60 report receiving pension, we adopt a Fuzzy RD design, which allows the jump in the probability of assignment to the treatment at the threshold to be smaller than 1. In this design, we interpret the ratio of the jump in the regression of the outcome on age to the jump in the regression of the treatment indicator on age as an average causal effect of the treatment (Imbens and Lemieux, 2008) This is analogous to an "intent to treat" parameter of a randomized controlled trial where the treatment is receiving pension income ( pension $_{i}$ ), but

\footnotetext{
${ }^{4}$ Since the labor market condition in 2009 is expected to be closer to 2011, it is further reassuring to find that the migration rate and its change around the age cutoff in 2009 is very similar to that in 2006, both are in the pre-NRPS period. Placebo test results using the 2009 wave are available upon request.
} 
because of "lack of compliance" a few people assigned to treatment by passing the eligible age $\left(1\left[\operatorname{age}_{i} \geq 60\right]\right)$ do not end up receiving pension. Formally, the estimand is

$$
\tau_{\mathrm{FRD}}=\frac{\lim _{a \downarrow 60} E\left[y \mid \operatorname{age}_{i}=a\right]-\lim _{a \uparrow 60} E\left[y \mid \operatorname{age}_{i}=a\right]}{\lim _{a \downarrow 60} E\left[\text { pension }_{i} \mid \operatorname{age}_{i}=a\right]-\lim _{a \uparrow 60} E\left[\text { pension }_{i} \mid \operatorname{age}_{i}=a\right]}
$$

To interpret this result, we further require that assignment to treatment satisfy a monotonicity property. Specifically, we are interested in how pension income impacts subsequent outcomes for compliers. Compliers are individuals who do not receive a pension if not eligible (below age 60) and receive a pension if eligible (above age 60). Formally,

$$
\tau_{\mathrm{FRD}}=E\left[y_{i}(1)-y_{i}(0) \mid \text { individual } i \text { is a complier and age } i_{i}=a\right]
$$

Following Lee and Lemieux (2010) and estimating variants of the following equations:

$$
\begin{gathered}
Y_{i}=\alpha_{1}+f_{1}\left(\text { age }_{i}-60\right)+1\left[\operatorname{age}_{i} \geq 60\right] \cdot \pi_{1}+\mathrm{X}_{i} \delta_{1}+\varepsilon_{1 i} \\
\text { pension }_{i}=\alpha_{0}+f_{2}\left(\operatorname{age}_{i}-60\right)+1\left[\operatorname{age}_{i} \geq 60\right] \cdot \pi_{0}+\mathrm{X}_{i} \delta_{0}+\varepsilon_{0 i}
\end{gathered}
$$

where $Y_{i}$ is the vector of outcome variables, pension $_{i}$ indicates whether a person actually receives pension, $f_{1}(\cdot)$ and $f_{2}(\cdot)$ are polynomials in the distance from the age 60 cutoff, $\mathrm{X}_{\mathrm{i}}$ is a vector of rich demographic and socioeconomic covariates of both parents and children, including ethnicity, cadre and party membership, education, marital status, years of marriage, religious beliefs, family assets. Besides, we control for adult child age, child birth order, and number of sons and daughters of parents, and village fixed effects. $\varepsilon_{i}$ is the error term. As long as $f_{1}(\cdot)$ and $f_{2}(\cdot)$ are continuous at $\operatorname{age}_{i}=60, \pi_{1}$ and $\pi_{0}$ represent the magnitude of the discontinuities in the numerator and denominator, respectively, of (1). $\hat{\tau}_{F R D}=\hat{\pi}_{1} / \hat{\pi}_{0}$ estimates the Local Average Treatment Effect (LATE) of pension receipt. The inclusion of covariates $X_{i}$, however, should not affect the estimation of $\pi_{1}$ and $\pi_{0}$ if $X_{i}$ do not vary discontinuously at the age cut-off. In Appendix Figure 2, we draw the relationships between main baseline covariates and normalized age to check discontinuity.

To overcome the challenge of finding an appropriate estimator for $f_{1}(\cdot)$ and $f_{2}(\cdot)$, we specify a flexible parametric model for $f_{1}(\cdot)$ and $f_{2}(\cdot)$. The main results in this paper adopt the linear specification since a model selection algorithm using the Schwarz (1978) criterion suggests that in more than half of all cases the preferred specification is linear. In Appendix Table 2, we present the main results using the $2^{\text {nd }}$ order and the $3^{\text {rd }}$ order polynomial specifications. Specifically, when, for example, a $3^{\text {rd }}$ order polynomial form is specified, $f_{1}(\cdot)$ and $f_{2}(\cdot)$ take the following forms 


$$
f_{1}\left(\text { age }_{i}-60\right)=1\left[\operatorname{age}_{i} \geq 60\right] \cdot \sum_{p=1}^{3} \gamma_{1 p}\left(\text { age }_{i}-60\right)^{p}+\left(1-1\left[\operatorname{age}_{i} \geq 60\right]\right) \cdot \sum_{p=1}^{3} \gamma_{1 p}^{\prime}\left(\text { age }_{i}-60\right)^{p}
$$

$$
f_{2}\left(\operatorname{age}_{i}-60\right)=1\left[\operatorname{age}_{i} \geq 60\right] \cdot \sum_{p=1}^{3} \gamma_{0 p}\left(\operatorname{age}_{i}-60\right)^{p}+\left(1-1\left[\operatorname{age}_{i} \geq 60\right]\right) \cdot \sum_{p=1}^{3} \gamma_{0 p}^{\prime}\left(\operatorname{age}_{i}-60\right)^{p}
$$

in which each term of the polynomial is interacted with the age eligibility dummy, allowing each term to have different parameters on either side of the age cutoff. $\gamma_{1 p}, \gamma_{1 p}$ and $\gamma_{1 p}^{\prime}, \gamma_{0 p}^{\prime}$ are coefficients on the $p$ polynomial terms for people eligible and ineligible for pension income, respectively, allowing the shape of the underlying conditional expectation to be different to the left and right of the threshold.

We first present the results of reduced form regressions that estimate the numerator and denominator in (2) respectively. To do so, we first use OLS regression for each of the estimations and then RD regression with different bandwidths. Next, we report the RD results of the treatment effect $\tau_{F R D}$.

All the RD estimates are bias-corrected with robust standard errors. The main results of this paper use the triangular kernel ${ }^{5}$ with the Calonico, Cattaneo, and Titiunik $(2014$, CCT hereafter) bandwidth selector. In Appendix Table 2, we also report results using the CrossValidation (CV hereafter) bandwidth selector (Imbens and Lemieux 2008), and the Imbens and Kalyanaraman (2012, IK hereafter) bandwidth selector. We also report main outcomes with half of the optimal bandwidth to increase the weights given to observations closer to the cut-off.

\section{Empirical Results}

\subsection{Summary statistics}

Appendix Figure 2 draws summary statistics for adult children as well as their parents. For individuals in both sub-samples, more than 80 percent are in the Han group, and the rest are ethnic minorities. Around 20 percent have religious beliefs, mainly Taoism. Some characteristics differ between the two sub-samples. Less than 5 percent adult children hold village leader positions and/or party membership, while this number is larger for elderly parents. Almost 40 percent of adult children finish nine-year mandatory education, while only 10 percent of parents do.

Table 1 presents summary statistics for adult children's migration status. Column (1) reports the mean and standard deviation for the whole sample. Columns (2) and (3) report means and standard deviations when confining the sample to adult children with parents between

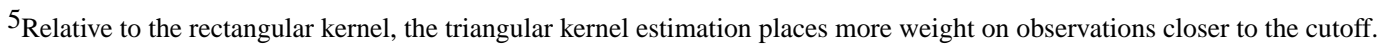


ages 55 and 65 , i.e. 5 years around the age cut-off. For each adult child in a family, we assign the running variable, age, in two scenarios. First, the younger age among parents is assigned if they are both older than age 60 . Second, the older age among parents is assigned if they are both younger than age 60 or if one of the two is older than age 60. Column (4) reports the result of $t$-test comparing the difference in adult children migration status between those below and above the pension eligibility threshold.

Traditionally, adult children, especially adult sons, provide care and assistance to their elderly parents in rural China. The aging trend of their parents may discourage adult children from migration. Therefore, the positive association between pension eligibility and migration rate are quite salient considering that life-cycle factors may work in the opposite direction that prevents us from observing this pattern.

Note that these descriptive statistics could be very different from the causal effect of pension receipt or the estimands in expression (1) and (2) as the means and t-tests may capture the impact of pension receipt as well as age and cohort heterogeneity. Fortunately, a carefully designed RD might be able to distinguish the discrete change associated with pension eligibility and the resulting pension payment from the life-cycle pattern of extended families. Next we fit a RD design to isolate the causal impact of pension income.

\subsection{Empirical Estimations}

A. The first stage: Pension receipt-Figure 2A plots the average rate of parents' pension receipt against the cut-off age 60 for the whole sample of adult children $(\mathrm{N}=1741)$. The fitted probability curve of receiving pension in Figure 2B suggests an almost 80 percentage jump in pension receipt immediately above the cut-off age.

We regress a binary indicator of receiving pension, allowing a change in pension eligibility at age 60. Panel A of Table 2 shows that around 77 percentage more people receive pension payment at the eligibility threshold. To make sure the discontinuity of pension receipt holds for all subsamples we analyze, Panel B through Panel E run first stage RD estimation for each subsample. Results suggest 70 to 86 percentage discontinuous jumps in the rate of pension receipt across all subsamples.

B. Migration decisions among adult children-Using the 2011 wave survey (our $4^{\text {th }}$ wave survey, post-NRPS period), we present the graphic results in Figure 3 and Figure 4 and numeric results of OLS, the reduced form RD, and RD LATE in Table 3. Estimations using the 2006 data (our $2^{\text {nd }}$ wave survey, pre-NRPS period) serve as a placebo. Since no rural pension program was implemented before 2010 in the surveyed region, we are confident that the discontinuity in 2011 is not driven by any pre-existing discrete change at age 60 if there is no discrete change at the age cut-off using the 2006 survey data.

Indeed, Figure 3 Panel A shows that adult children's migration rate in 2011 is more constant before the cutoff, jumps immediately afterwards, and then drops down rapidly. ${ }^{6}$ However, we do not observe a similar pattern in Panel B using the 2006 data. ${ }^{7}$ Consistently, the estimands in Table 3 show a statistically significant jump in migration but no discrete change in migration status before the NRPS, which lends us credibility of the results. 
Sons may respond to pension receipt of their parents differently from daughters do. Figure 4 separately plots the migration rate around the age cutoff for adult sons (Panel 4A) and adult daughters (Panel 4B). We then repeat the exercise in Table 3 separately for adult sons (Panel B) and adult daughters (Panel C) and find a significant jump in migration only for adult sons. Though remains insignificant, the effect for adult daughters is more precisely estimated when richer covariates are included. Some of these covariates are worth discussing. First, we control for marital status as married daughters may respond less than unmarried daughters to their parents' pension receipt. Second, daughters married earlier might be subject to a stronger patriarchal norm. Though we are less concerned about this issue due to the fact that the patriarchal norm has been evolving over hundreds of years and that the norm is rather homogeneous in our surveyed region, we control for years of marriage for children to capture the potential patriarchal trend. Third, we control for birth order, since daughters of higher birth order may react differently from younger siblings. For example, they are often more responsible for taking care of parents and sharing household chores. Fourth, we also control for number of sons and number of daughters as family size and composition may also matter to children's reaction to parental pension receipt. Finally, there is, to the best of our knowledge, no law or customs regarding differential rights to inheritance between sons and daughters.

In Table 3 Panel D and Panel E, we categorize the sample by self-rated health status of parents. Verified that family economic resources are smooth around the age 60 threshold, we hypothesize that when any elderly parent is in poor health, adult children may stay to offer instrumental support and react less to the pension income. We indeed find that the effect is insignificant and of smaller magnitude for adult children with any parent in poor health, while a significant and larger effect is found for those with all parents in good health.

C. Additional Results-Our main estimations do not differentiate between families with both parents and families with only one parent. It is likely that having only one parent may significantly change the costs and benefits of adult children migration. For parents in the age range 55-65, only 59 adult children in our sample have only one parent. We are not able to implement a separate RD design for this very small number of cases around the threshold. However, we are able to re-estimate the main RD results by excluding those with one parent. Results, presented in the Appendix Table 3, show only slight changes in the marginal effects.

\footnotetext{
${ }^{6} \mathrm{~A}$ few points may worth mentioning about this pattern. First, the most credible part of RD design is comparisons very close to the cutoff age. The further away from the cutoff, the less reliable the statistical inference becomes. For example, some age related trend may confound the pension effect when we compare people more distant in ages. Second, in rural China life expectancy at birth is below 70, especially in the impoverished Guizhou province. Only 4.38 percent of respondents in our Guizhou sample are above age 70, making the identifications below age 70 more reliable. Third, the migration trend for the oldest of the old is quite similar between Panel 3A and Panel 3B. Much of the sharply down-moving part of the migration curve falls into this age group. This may be because children tend to be back to provide old-age support to parents as they get older.

${ }^{7}$ While there is no statistically significant discontinuity round the cutoff, we do observe that adult children's migration rate drops sharply when parents are younger than age 60 and rises back to the previous level after they become 60 . Since there was no social welfare program with an age 60 cutoff in 2006, this pattern is not likely to be explained by the changes in parents' financial uncertainty. Moreover, with any pension reform, children's potential anticipation effect should to some extent smooth this pattern. Even if the pension reform is completely out of expectation, it is more reasonable that they gradually move back due to the uncertain financial future for parents. Fortunately, the same placebo text using the 2009 wave results in much smoother migration rate around the cutoff, suggesting that sharp move-in and move-out only applies to the year 2006. We leave this remaining concern to our future research.
} 
In future research with a larger sample size, it would be valuable to test any differential effects of pension receipt by the number of parents.

Migration decisions of children may depend on their composition, including gender and number of siblings. For example, adult children with no siblings may behave vastly different from those with siblings. Ideally, it is best to restrict the analysis to households with same number of siblings and gender composition. The RD design recommends a large number of observations around the age threshold to better approach causal inference, while our survey, conducted in rural western China where the family planning policy has been loosely implemented, has only a small proportion (3.9 percent) of parents around ages $[55,65]$ with only one child. ${ }^{8}$

Though this small sample size prevents us from separately identifying changes in children's migration behavior for families with all different child gender and number compositions, we are able to estimate some of them. First, results comparing the subsample of daughters with brother(s) versus those without a brother suggest a slightly higher but still insignificant jump in daughters' migration in the former subsample. Daughters might be more confident to move if they have brother(s) who follow the traditional norm to provide old-age support to parents. However, their decisions to migrate may still rely on their husbands' families. Second, results from the majority of households with more than one child remain.

Overall, we should not worry about sibling composition as a confounder of pension effect. In the RD design, parents barely below the age cut-off should have statistically similar composition of children compared with parents barely above the age cut-off. Running three $\mathrm{RD}$ regressions respectively using number of children, number of sons, and number of daughters as outcome variables, there is no statistically significant difference in these outcomes as a result of pension receipt, reassuring us that household composition is not likely to confound the pension impact.

\subsection{Potential Mechanisms}

First of all, pension payment to elderly parents raise their income. Since work activities and income gradually decline as people age, an overall positive income shock is necessary to generate the observed pattern of adult child migration. Tests using our Guizhou survey reassure us that personal income of the elderly (excluding pension benefit) is continuous around the pension eligibility cutoff but discontinuously increases when pension income is accounted for. ${ }^{9}$

According to the policy, receiving pension payment from the NRPS is not tied to individual labor force participation. Similar to other parts of rural China, very small proportion of people (less than 2 percent in our sample) between ages 55-65 have a formal job. However, in rural China most elderly people remain active in agricultural production well into old ages, which becomes part of their lifestyle. In the 2011 wave survey, we collected

\footnotetext{
${ }^{8}$ This is consistent with main sources of population statistics in rural China, such as China General Social Survey (CGSS), China Population Census, and China Family Panel Studies (CFPS).

${ }^{9}$ Using two national samples, China Health and Retirement Longitudinal Study (CHARLS) and China Family Panel Studies (CFPS), Zhang et al. (2013) and Chen and Wang (2014) also find largely continuous income profile around the cut-off age.
} 
information on time use in the farm for each individual. Both graphic results and nonparametric RD tests of four indicators of agricultural labor participation among rural Guizhou residents, including whether work in the farm, number of farming months per year, number of farming days per month in busy season, and number of farming hours per month in busy season, all suggest continuities around age 60 . Results are available upon request.

As half of the surveyed households are still below the USD 1.25 international poverty line (Appendix Table 1), pension payment may provide parents in these extended families more economic resources that they can stand on their feet. As a result, their adult children may be freed up to a larger feasible set of occupational choices via migrating from traditional rural community to seek better jobs in modernized urban industries. Figure 1 indicates that a majority of adult children take off-farm jobs after migration.

We observe much stronger motivation among adult sons (but not daughters) to leave the home county upon their parents receiving pension payment (Figure 4). First, a large proportion of marriages are across counties. The patriarchal system in China encourages daughters to live with husbands' families after getting married, while married sons and daughters-in-law often stay with sons' parents in the home villages. This tradition may explain why adult daughters have an overall higher rate of migration than adult sons. Meanwhile, because of the patriarchal system, adult sons may respond more to their parents' more economic independence as a result of pension. In comparison, married daughters may be less responsive to pension receipt of their own parents than that of their husbands' parents. Therefore, we see a larger and more significant jump in migration around the pension eligibility cut-off for sons. In addition, migrant sons are more likely to move back to the home county to take care of parents when they are getting old, while migrant daughters are less responsive to the aging of their own parents. Therefore, we observe a sharper decline in migration rate for sons than daughters when their parents are getting older. All above observations are consistent with the fact that daughters play a less important role in providing care to their parents under the patriarchal residency tradition in rural China.

Some other potential channels deserve further research when appropriate data becomes available. First, the impact of pension income might be partially attributable to the increase in household resources it represents, which can be used to stake migrants until they become self-sufficient. Further, if income from off-farm jobs is subject to large fluctuations, an increase in income of pensioners may serve as insurance that enables their adult children to endure larger income risks. To investigate this potential channel, extremely rich information on intergenerational financial transfers between elderly parents and (resident and nonresident) adult children as well as potential risks and benefits for categories of off-farm jobs matched to specific immigrant urban areas are required.

Second, part of the impact may originate from the presence of pensioners who can care for small children, which allows prime-aged adults to look for work elsewhere. A test of this potential channel requires rich data on intergenerational transfers and care offered by grandparents to grandchildren. 


\section{Robustness}

This section presents several pieces of evidence that key identification conditions for RD design are met. First, we test possible discontinuities at other age cut-offs, which sheds light on whether the jumps we observe are driven by other age-related changes rather than the arrival of pension income. An individual barely below pension eligible age should be very similar to those just turn 60 except that the former receives no pension payment. Table 4 reports the placebo test results on pension receipt and adult children migration using age 59 as the cut-off. The first stage estimation of pension receipt finds no significant change around the placebo age cut-off. First stage placebo tests using other subsamples, available upon request, are quite similar. In the second stage, the RD estimands show no significant pattern for adult children migration. The same tests are conducted for other placebo age cutoffs nearby, such as 59.5, 60.5 and 61, again no significant discontinuities are found. Therefore, we are confident that the discrete change in migration status at age 60 is driven by pension income.

If people can borrow from future, they may do so, for example, by saving less or borrowing more from others in anticipation of an elderly parent becoming eligible for pension income in the future. ${ }^{10}$ The anticipation effect, if exists, may offset the impact of pension, rendering our identified pension impact a lower bound of the true effect of pension on adult children migration. The evidence of no jump at the placebo cut-off ages 59 and 59.5 before the real cut-off age 60 suggests no such anticipation effect and implies that the households are constrained to borrow.

Second, we test for discontinuities in baseline characteristics that should not be affected by the treatment. If baseline demographic or socioeconomic characteristics show discontinuous jumps, we would be concerned about the similarity between people barely below and barely above the age cut-off. While we can never be certain that the unobservable characteristics of the elderly satisfy this condition, the validity of this assumption can be tested by ensuring that the conditional expectations of the observable characteristics do not vary discontinuously in the neighborhood of the cut-off age. Appendix Figure 2 shows the graphic equivalent of the test on main baseline demographic and socioeconomic characteristics of parents (Appendix Figure 2b) and their adult children (Appendix Figure 2a). All the horizontal axes represent parents' normalized age relative to 60 . The vertical axes are ethnicity, cadre and party membership, whether one finishes nine-year mandatory education, marital status, and religious beliefs, respectively. Graphic tests on other covariates, i.e. different types of household assets, are not reported. Though children's marital status, religious beliefs, and ethnicity do not seem to be as smooth as other baseline characteristics over the pension age cutoff, our nonparametric RD results for these characteristics reported below the figures, including coefficients, standard errors, and optimal bandwidth automatically chosen, suggest that none is statistically discontinuous around the cut-off. Furthermore, there should be little concern about significant

\footnotetext{
${ }^{10}$ The elderly often have limited ability to borrow from future given their declining income as they age. Though the pension benefit only accounts for a small proportion of household income, it means a 7-8 times higher income for the elderly population in rural China who sometimes had no income before the NRPS (Zhang et al. 2013).
} 
discontinuities since the benefit of enrolling in the NRPS does not rely on children's ethnicity, marital status or religious reliefs. The latter two characteristics are predetermined and rarely change. The remaining concerns are mitigated through controlling for all these observable characteristics in the RD design.

Third, another standard check in a RD design is to verify that no other predetermined outcomes display discontinuities around the cut-off apart from the treatment. As already reported in Table 3, outcome variables before the pension program, drawn from our 2006 wave survey, find no discrete change at the age cut-off, which lends us credibility that our results should not be driven by these predetermined jumps at the cut-off.

Fourth, we test for continuity of age density. Assignment to pension program around the age 60 threshold should ideally be randomized. However, if richer families are more able to manipulate age, they are more likely to receive pensions and be able to support their children to migrate. This could lead to biased estimates of the effect of pension payment. In the present case, however, this seems unlikely. The age profiles on the registration card were issued far ahead of the pension reform. Moreover, China's strict hukou system mitigates the potential cases of misreporting age. The normalized age as a continuous running variable further eliminates the heaping issue as a result of irregular rounding up (or down) of age profile.

Formally, we conduct two verification tests. First, we examine the income and other characteristics of the very few cases in our sample who report receiving pensions before age 60. No significant demographic and socioeconomic characteristics are found for this small group of pensioners. Second, we check whether there is a discontinuity of age profiles at the age cut-off - in this case whether "missing" ages just below the cut-off are followed by "hump" above. The histogram of age in Appendix Figure 3 shows little discrete change in the age density around the age 60 cut-off. We adopt the McCrary (2008) test for discontinuity in the age density function. We also fit a linear term in the normalized age, a dummy equals one if one is eligible for pension income, and their interaction to the log of the fraction of observations with each baseline age using weighted least squares regression. The test confirms that no statistically significant discontinuities are evident in the (log of the) age density.

Fifth, to our knowledge, there exists no other policy discontinuity at age 60 that may confound the identification of pension receipt. For example, there is no other social security program stipulating that people above age 60 are eligible for it. Meanwhile, there is no subsidized nursing home regulating that age 60 is the eligible age cut-off to live in and therefore enable adult children to migrate.

Finally, besides the CCT bandwidth selector, our results are robust to other bandwidth selection procedures, such as the CV bandwidth selector and the IK bandwidth selector (Appendix Table 2). Results are also robust to bandwidth selections. The optimal bandwidth for our RD design is around 2 years. Further, main outcomes with half of this optimal bandwidth (1 year) and with twice of this optimal bandwidth (4 years) generate quite similar 
results (Appendix Table 2). Results are also robust to polynomial selections, such as in linear, quadratic and cubic specifications (Appendix Table 2).

\section{Conclusions}

This paper provides early evidence on the impact of the newly implemented old-age pension program, the NRPS in China, on migration decisions among adult children. Utilizing our primary longitudinal household survey from rural western China, we show that adult children, who by virtue of having parents reach age 60 and receive pension, are more likely to migrate out of their home county to pursue off-farm jobs.

We contribute to the growing body of research indicating the large impact of social pension on pensioners and its spillover effects on extended families, especially providing the first piece of evidence for China where the new pension payment is not as generous. More importantly, this paper is among the first that apply RD in the study of social pension, which overcomes two main challenges in the literature that pension eligible households can be quite different from ineligible households and that age or cohort related heterogeneity may confound the pension impact.

Our empirical findings hold important implications and general lessons for household theory. First, social pensions directed at the elderly could benefit other generations. Second, pension programs that transfer income to elderly population should be more cautious as substantive changes in child migration may offset some benefits through reduced companionship and instrumental support to parents provided by children. Third, the changes in adult children's migration as a result of pension payment may have implications for other aspects of family life that deserve more research.

Future research directions involve testing whether gender identity of the pension recipient affects its use for migration. Pension payment provides an income source that is attributed to a specific family member and can be used to assess how household behavior and well-being are affected differently due to different members gaining control of this income source. Future studies will distinguish potentially differential impacts of income shocks to fathers versus mothers.

\section{Supplementary Material}

Refer to Web version on PubMed Central for supplementary material.

\section{Acknowledgments}

Financial support from the NIH/NIA (Grant No. 1 R03 AG048920-01) and the Natural Science Foundation of China (NSFC) (Approval Nos. 70525003 and 70828002) and data collection support provided by IFPRI, China Academy of Agricultural Sciences, and Guizhou University are acknowledged. I am grateful to Kevin Chen for providing the latest wave (2012) Guizhou survey data. I also wish to thank Joe Altonji, Karen Eggleston, Jason Fletcher, and Jody Sindelar for helpful comments and discussions. Seminar audiences at the IZA workshops, AEA annual meetings, the NBER Summer Institute, University of Connecticut and Yale University are acknowledged. The views expressed herein and any remaining errors are the author's and do not represent any official agency. 


\section{References}

Ardington C, Case A, Hosegood V. Labor Supply Responses to Large Social Transfers: Longitudinal Evidence from South Africa. American Economic Journal: Applied Economics. 2009; 1(1):22-48. [PubMed: 19750139]

Bertrand M, Mullainathan S, Miller D. Public Policy and Extended Families: Evidence from Pensions in South Africa. World Bank Economic Review. 2003; 17(1):27-50.

Calonico S, Cattaneo MD, Titiunik R. Robust Data-Driven Inference in the Regression-Discontinuity Design. The Stata Journal. 2014; 14(4):909-946.

Card, David, Dobkin, Carlos, Maestas, Nicole. Does Medicare Save Lives? The Quarterly Journal of Economics. 2009; 124(2):597-636. [PubMed: 19920880]

Chen X, Wang T. Old-Age Pension, Subjective Well-being and Mental Health: Evidence from the New Rural Pension Program in China using A Regression Discontinuity Design. Paper presented in the NBER Summer Institute. 2014; 2014

Edmonds, Eric, Mammen, Kristin, Miller, Douglas L. Rearranging the family? Income support and elderly living arrangements in a low income country. Journal of Human Resources. 2005; 40(1): 186-207.

Imbens GW, Kalyanaraman K. Optimal Bandwidth Choice for the Regression Discontinuity Estimator. Review of Economic Studies. 2012; 79(3):933-959.

Imbens, Guido, Lemieux, Thomas. Regression discontinuity designs: A guide to practice. Journal of Econometrics. 2008; 142(2):615-635.

Jensen, Robert T. Do private transfers 'displace' the benefits of public transfers? Evidence from South Africa. Journal of Public Economics. 2004; 88(1-2):89-112.

Lee, David S., Lemieux, Thomas. Regression Discontinuity Designs in Economics. Journal of Economic Literature. 2010; 48(2):281-355.

Lund, Frances. State Social Benefits in Social Africa. International Social Security Review. 2007; 46(1):5-25.

McCrary, Justin. Manipulation of the Running Variable in the Regression Discontinuity Design: A Density Test. Journal of Econometrics. 2008; 142(2):698-714.

Miller G, Pinto D, Vera-Hernandez M. Risk protection, service use, and health outcomes under Colombia's health insurance program for the Poor. American Economic Journal: Applied Economics. 2013; 5(4):61-91. [PubMed: 25346799]

Posel D, Fairburn J, Lund F. Labor Migration and Households: A Reconsideration of the Impact of Social Pension on Labor Supply in South Africa. Economic Modelling. 2006; 23(5):836-853.

Schwarz G. Estimating the dimension of a model. The Annals of Statistics. 1978; 6:497-511.

Sun A, Eggleston K. The impact of rural pensions in China on migration and off-farm employment of adult children and extended households' living arrangement. 2014 unpublished working paper.

Zhang C, Giles J, Zhao Y. Policy Evaluation of China's New Rural Pension Program: Income, Poverty, Expenditure, Subjective Wellbeing and Labor Supply (in Chinese). CCER Working Paper. 2013 
A

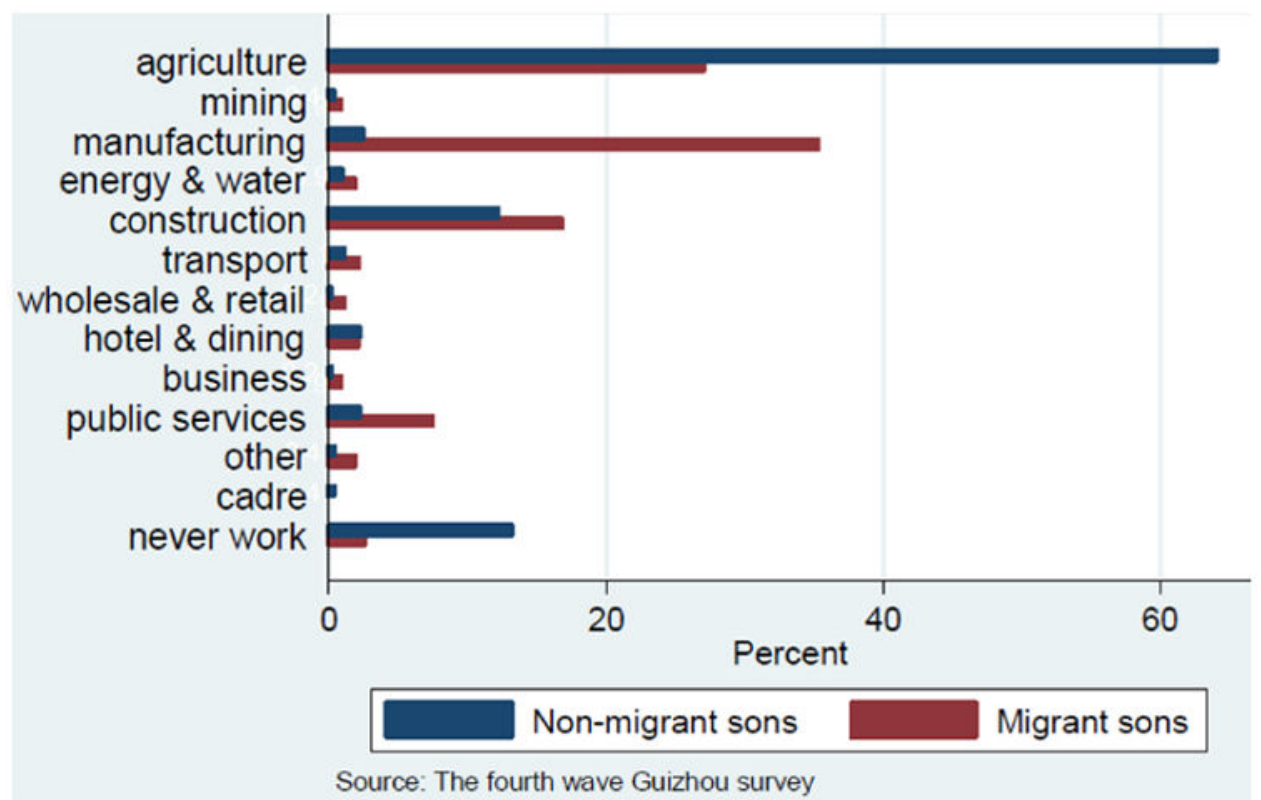

B

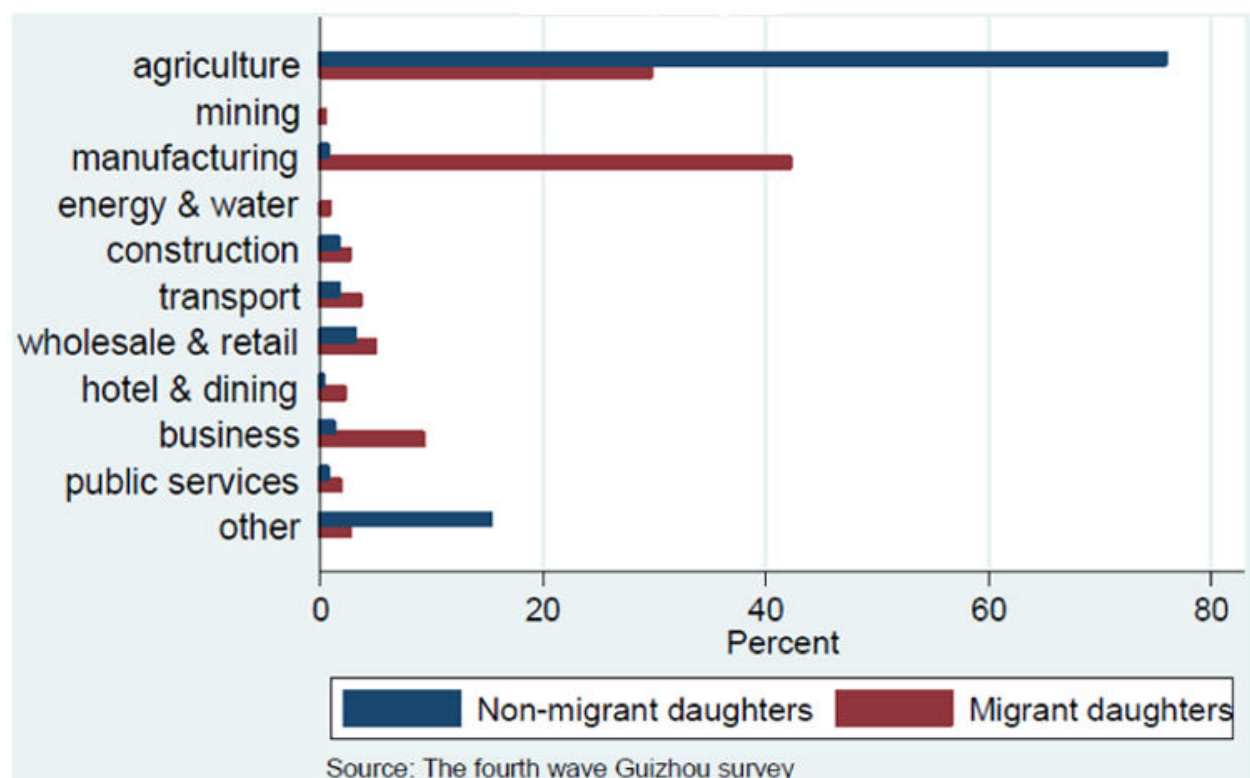

Figure 1. A. Occupational choices among adult sons by migration status, B. Occupational choices among adult daughters by migration status 


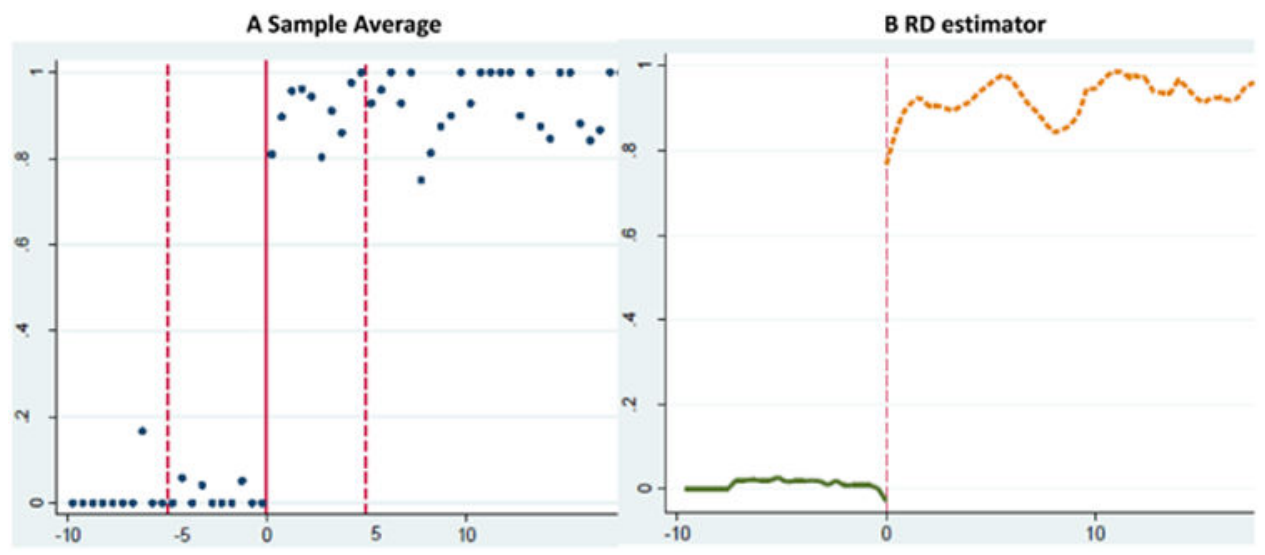

Figure 2. Parents' pension receipt according to normalized age $(0=$ eligibility threshold at age 60$)$ Source: The fourth wave (2011) Guizhou survey.

Notes: This figure shows the relationship between rate of pension receipt and age. Age is normalized based on the day, month, and year information from date of birth.

General graphing notes: $0=$ eligibility threshold at age 60 . The lines are nonlinear fit using triangular weights on either side using the micro data. The dots represent averages of bins centered at .5 year bins (approximately 180 days). Graphs with different window and bin widths are available upon request. 
Panel 3A. Post-NRPS RD Estimator (2011)

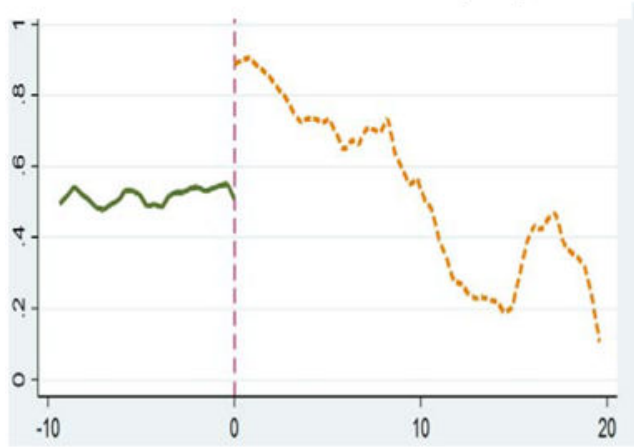

Panel 3B. Pre-NRPS RD Estimator (2006)

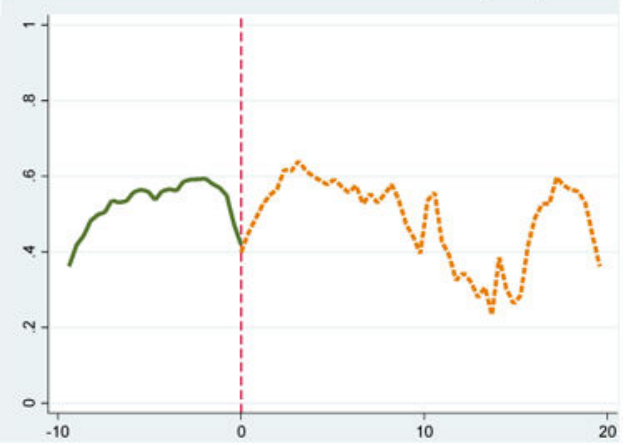

Figure 3. Migration status of adult children, for treatment (2011) and placebo (2006) years Source: The second wave (2006) and the fourth wave (2011) Guizhou survey.

Notes: This figure shows the relationship between migration rate of adult children and normalized age of the older parent. General notes from Figure 2 apply. 
Panel 4A. RD Estimator for sons (2011)

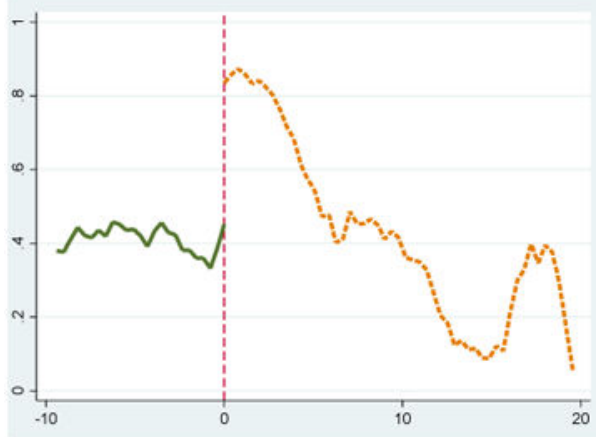

Panel 4B. RD Estimator for daughters (2011)

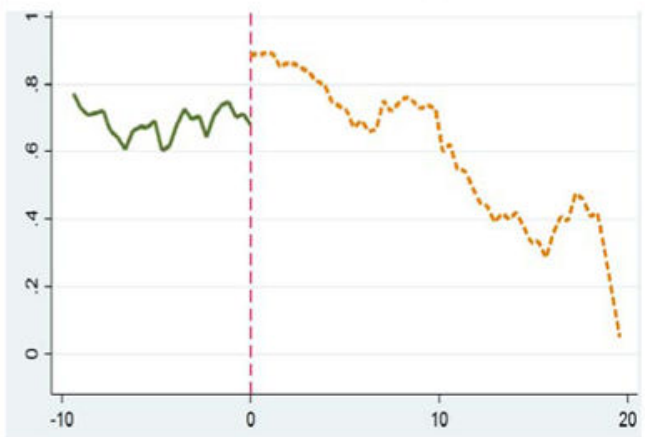

Figure 4. Migration status of adult children by gender Source: The fourth wave (2011) Guizhou survey.

Notes: This figure shows the relationship between migration rate of adult children and normalized age of the older parent. General notes from Figure 2 apply. 
Table 1

Summary statistics for migration status (Sample of adult children, $N=1741$ )

\begin{tabular}{lcccc}
\hline Dependent variable & All & {$[-\mathbf{- 5}$ yrs,0) } & {$[\mathbf{0 , 5}$ yrs $]$} & Diff (3)-(2) \\
\hline $\mathrm{N}$ & $\mathbf{( 1 )}$ & $\mathbf{( 2 )}$ & $\mathbf{( 3 )}$ & $\mathbf{( 4 )}$ \\
\hline Indicator of migration & $.401(.490)$ & $.432(.491)$ & $.703(.391)$ & $.271 * * *(.035)$ \\
\hline
\end{tabular}

Source: The fourth wave (2011) Guizhou survey.

Notes: $[-5 \mathrm{yrs}, 0)$ and $[0,5 \mathrm{yrs}]$ mean parents' age relative to the $60 \mathrm{yrs}$ cutoff. $\mathrm{N}$ denotes sample size. 


\section{Table 2}

\section{First Stage: Parental pension receipt}

\begin{tabular}{|c|c|c|c|}
\hline Dependent variable & $\mathbf{N}$ & OLS & RD estimate \\
\hline & (1) & (2) & (3) \\
\hline \multicolumn{4}{|c|}{ Panel A. Sample of adult children $(N=1741)$} \\
\hline Indicator of parental pension receipt & 1741 & $.932^{* * *}(.021)$ & $.766^{* * *}(.131)$ \\
\hline \multicolumn{4}{|c|}{ Panel B. Sample of adult sons $(N=1016)$} \\
\hline Indicator of parental pension receipt & 1016 & $.924^{* * *}(.019)$ & $.861^{* * * *}(.049)$ \\
\hline \multicolumn{4}{|c|}{ Panel C. Sample of adult daughters $(N=725)$} \\
\hline Indicator of parental pension receipt & 725 & $.899^{* * *}(.027)$ & $.701^{* * *}(.113)$ \\
\hline \multicolumn{4}{|c|}{ Panel D. Sample of adult children with a parent in poor health $(N=970)$} \\
\hline Indicator of parental pension receipt & 970 & $.897^{* * *}(.054)$ & $.765^{* * *}(.117)$ \\
\hline \multicolumn{4}{|c|}{ Panel E. Sample of adult children with all parents in good health $(N=771)$} \\
\hline Indicator of parental pension receipt & 771 & $.871^{* * * *}(.037)$ & $.857^{* * * *}(.123)$ \\
\hline
\end{tabular}

Source: The fourth wave (2011) Guizhou survey.

Notes:

[1] The optimal bandwidths are calculated implementing the CCT bandwidth selector.

[2] The nearest-neighbors match procedure is implemented to compute the variance-covariance matrix estimator.

[3] The RD regression is weighted using triangular weights. The RD estimates are bias-corrected with robust standard errors.

[4] The regressions control for very rich characteristics of both parents and children, including ethnicity, cadre and party membership, education, marital status, years of marriage, religious beliefs, family assets. Besides, we control for adult child age, child birth order, and number of sons and daughters of parents, and village fixed effects.

\footnotetext{
Significant at the 1 percent level.

*** Significant at the 5 percent level.

*

Significant at the 10 percent level.
} 


\section{롤 \\ ㄱ.}

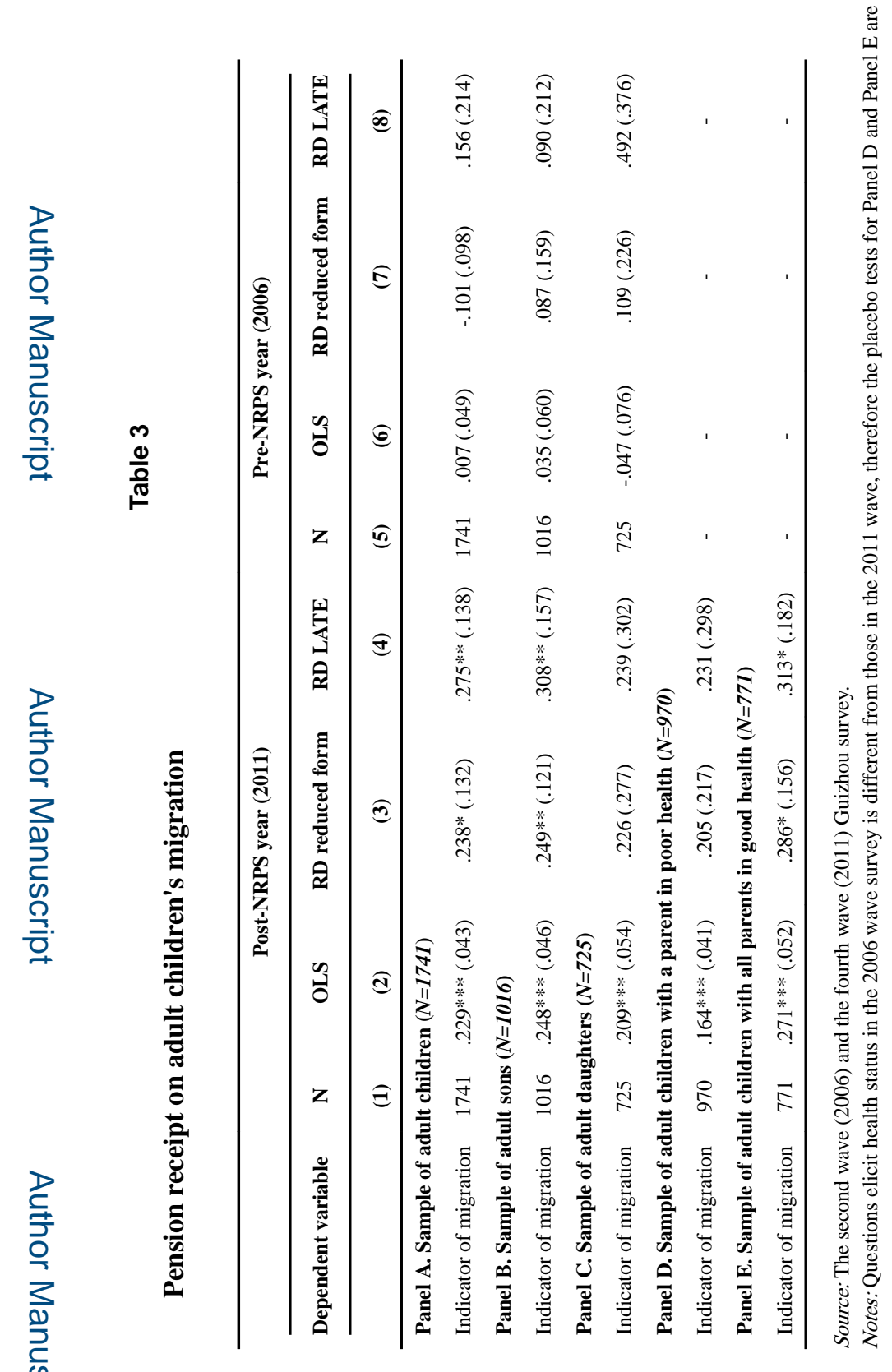

Contemp Econ Policy. Author manuscript; available in PMC 2017 October 01. 
Table 4

Effect on main outcomes allowing changes at age 59 (Sample of adult children, $N=1741$ )

\begin{tabular}{lcccc}
\hline Dependent variables & $\mathbf{N}$ & OLS & RD reduced form/first stage & RD LATE \\
\hline & $(\mathbf{1})$ & $(\mathbf{2})$ & $(\mathbf{3})$ & $(\mathbf{4})$ \\
\hline Indicator of parental pension receipt & 1741 & $.198(.253)$ & $-.046(.119)$ & - \\
\hline Indicator of migration & 1741 & $.185^{*}(.106)$ & $-.118(.139)$ & $.151(.287)$ \\
\hline
\end{tabular}

Source: The fourth wave (2011) Guizhou survey.

Notes: The tests using other subsamples also generate insignificant effect of pension receipt around age 59 placebo cut-off.

General notes from Table 2 apply. 